\title{
A FRAMEWORK FOR RESPONSE SURFACE METHODOLOGY FOR SIMULATION OPTIMIZATION
}

\author{
H. Gonda Neddermeijer \\ Econometric Institute and \\ Department of Public Health \\ Erasmus University Rotterdam \\ P.O.Box 1738 \\ 3000 DR Rotterdam \\ THE NETHERLANDS
}

\author{
Gerrit J. van Oortmarssen \\ Department of Public Health \\ Erasmus University Rotterdam \\ P.O.Box 1738 \\ 3000 DR Rotterdam \\ THE NETHERLANDS
}

\author{
Nanda Piersma \\ Rommert Dekker \\ Econometric Institute \\ Erasmus University Rotterdam \\ P.O.Box 1738 \\ 3000 DR Rotterdam \\ THE NETHERLANDS
}

ECONOMETRIC INSTITUTE REPORT EI2000-14/A

Keywords: Optimization; Simulation

\begin{abstract}
We develop a framework for automated optimization of stochastic simulation models using Response Surface Methodology. The framework is especially intended for simulation models where the calculation of the corresponding stochastic response function is very expensive or time-consuming. Response Surface Methodology is frequently used for the optimization of stochastic simulation models in a non-automated fashion. In scientific applications there is a clear need for a standardized algorithm based on Response Surface Methodology. In addition, an automated algorithm is less timeconsuming, since there is no need to interfere in the optimization process. In our framework for automated optimization we describe all choices that have to be made in constructing such an algorithm.
\end{abstract}

\section{INTRODUCTION}

When optimizing a stochastic simulation model, one tries to estimate the model parameters that optimize specific stochastic output of the simulation model. In this optimization procedure, the simulation model is often considered as a black-box model (Pflug, 1996) where the output of the simulation model can be regarded as a stochastic function of the model parameters. In this paper we propose a framework for automated Response Surface Methodology (RSM) for the optimization of stochastic simulation models. This framework that is especially intended for simulation models where the calculation of the corresponding stochastic objective function is very expensive or time-consuming (Wright, 1996). The simulation models that we consider only have real-valued parameters in the optimization.

RSM is a collection of statistical and mathematical techniques useful for optimizing stochastic functions (Myers and Montgomery, 1995). It is frequently used for the optimization of stochastic simulation models (Fu, 1994; Carson and Maria, 1997; Kleijnen, 1998). This methodology is based on approximation of the stochastic objective function by a low order polynomial on a small subregion of the domain. The coefficients of the polynomial are determined by regression analysis applied to a number of observations of the stochastic objective function. To this end, the objective function is evaluated in an arrangement of points referred to as an experimental design (Kleijnen, 1998). Based on the fitted polynomial, the local best point is derived, which is used as a current estimator of the optimum and as the center point of a new region of interest $(\mathrm{Fu}, 1994)$, 
where again the stochastic objective function is approximated by a low order polynomial.

There is a vast amount of papers and books on RSM. For extensive information on various aspects of RSM we refer to Box and Draper (1987), Myers and Montgomery (1995) and Khuri and Cornell (1996). Hood and Welch (1993) give an outline of RSM when applied to non-automated optimization of simulation models. In non-automated optimization RSM is an interactive process in which one gradually gains understanding of the nature of the stochastic objective function. Based on these insights the algorithm can be adapted during the optimization exercise. In an automated RSM algorithm human intervention during the optimization process is of course not possible. A good automated RSM algorithm should therefore include some degree of self-correction mechanisms (Box and Liu, 1999).

The establishment of a clear and consistent RSM optimization algorithm is of significant importance for its use as a tool in scientific applications, e.g. for estimation of model parameters, where results should be reproducible and derived via a clear method. A complete and clear definition of all steps and choices in a RSM algorithm is also necessary for automated optimization where all choices concerning the algorithm have to be made at the outset of an application. Automated optimization is less time-consuming, since there is no need to interfere in this optimization process. This is an advantage in large-scale time-consuming applications. However, there is no consensus about a standard RSM algorithm.

For the optimization of stochastic simulation models several methods can be used, such as RSM, the Nelder and Mead simplex method (Neddermeijer et al., 1999) and Simultaneous Perturbation Stochastic Approximation (Fu and Hill, 1996). There are surprisingly few papers that systematically compare the performances of these optimization methods. Such a comparison clearly requires a standardized RSM algorithm.

Smith (1976) was the first to describe an automated optimum-seeking RSM program, without elaborating on the choices that are made within the RSM algorithm used in this program. Joshi, Sherali and Tew (1998) describe an enhanced algorithm for RSM and compare this algorithm with a standard RSM algorithm, again without describing this standard algorithm in detail. In this paper we will propose a framework for a Response Surface Methodology algorithm for automated simulation optimization. It will be obvious that this framework can also be used for non-automated optimization. We will discuss the choices that have to be made to construct a standard RSM algorithm and will mention relevant references on the choices concerned.

\section{THE FRAMEWORK}

Without loss of generality, we assume that the optimization is a minimization problem. Mathematically, this problem can be described by

$$
\text { minimize } f: D \rightarrow \mathbb{R}, D \subseteq \mathbb{R}^{k}
$$

where $f\left(\xi_{1}, \ldots, \xi_{k}\right)=\mathbb{E}\left(F\left(\xi_{1}, \ldots, \xi_{k}\right)\right)$ Here, $F\left(\xi_{1}, \ldots, \xi_{k}\right)$ denotes the stochastic output for given input $\left\{\xi_{1}, \ldots, \xi_{k}\right\}$, and $\mathbb{E}\left(F\left(\xi_{1}, \ldots, \xi_{k}\right)\right)$ denotes its expected value. When optimizing a simulation model, the arguments $\xi_{1}, \ldots, \xi_{k}$ represent the parameters of the simulation model. In RSM, the parameters of the simulation model are usually called factors, whereas the stochastic output is called the response of the simulation model. It is assumed that a screening phase, in which factors that are considered unimportant are eliminated from the optimization problem, as well as possible transformations of the factors and the response have already taken place.

Usually, a RSM algorithm comprises two phases. In the first phase the response surface function is approximated by first-order polynomials, until a polynomial is fitted that shows significant lack-of-fit, or until there is no direction of improved response anymore (Cochran and Cox, 1962). In the second phase the objective function is approximated by a second-order polynomial ( $\mathrm{Fu}$, 1994). On the basis of the various extensions and modifications of this classic algorithm that can be found in literature, we constructed a framework for an automated RSM algorithm, see Figure 1. The various elements of this framework are described in the remainder of this paper. 
Start

A) Approximate the response surface function locally by a

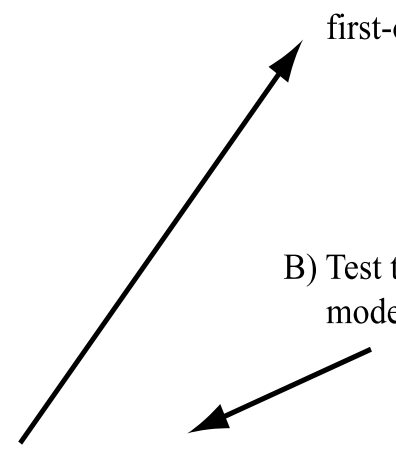
first-order model

C) Perform a line search in the steepest descent direction
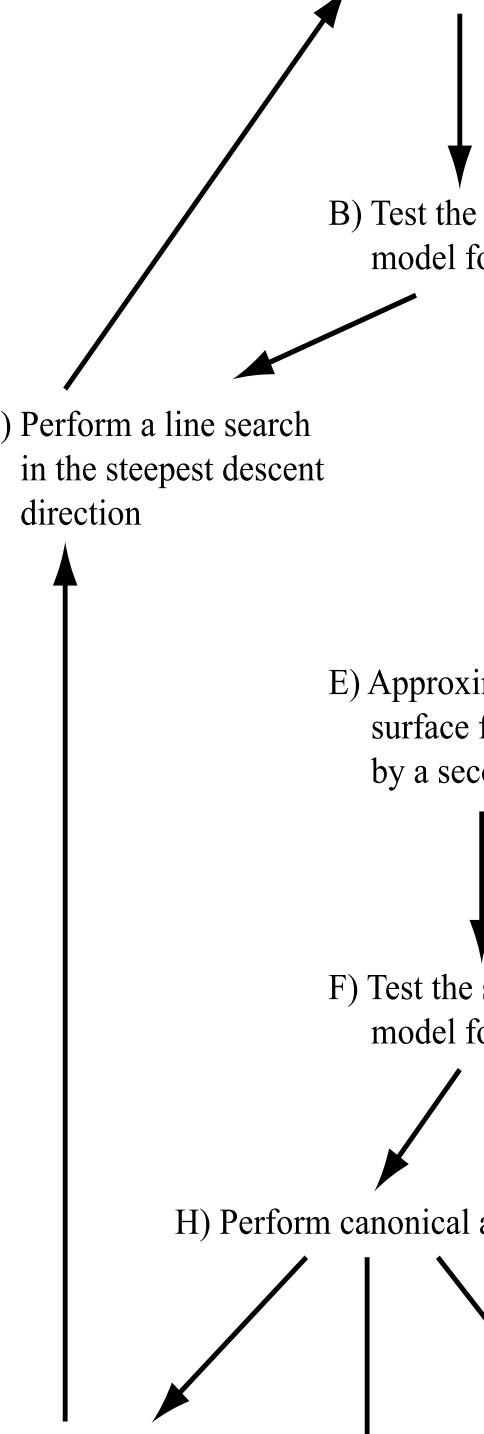

) Test the first-order model for adequacy

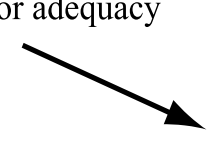

D) Solve the inadequacy of the first-order model

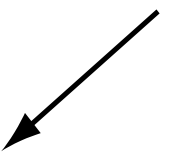

E) Approximate the response surface function locally by a second-order model

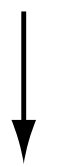

F) Test the second-order model for adequacy

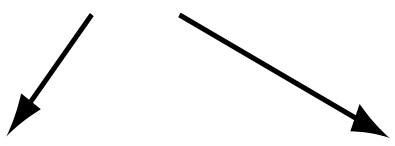

H) Perform canonical analysis
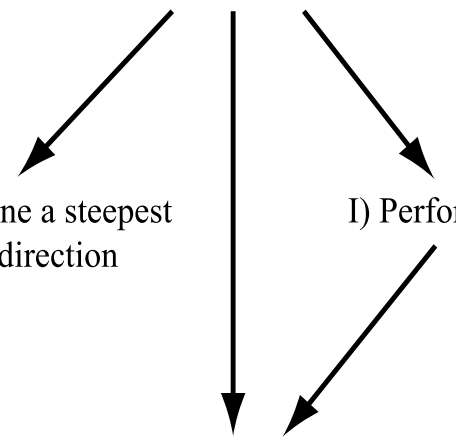

G) Solve the inadequacy of the second-order model

K) Determine a steepest descent direction

J) Accept the stationary point as the center point of the new region of interest

Figure 1: Framework for an automated RSM algorithm 
In each iteration $n, n \geq 1$, we consider a small subregion of the domain, which is called the region of interest and is denoted by

$$
\left[l_{1}^{n}, u_{1}^{n}\right] \times \ldots \times\left[l_{k}^{n}, u_{k}^{n}\right]
$$

This region can be described by a center point

$$
\xi_{i}^{n}=\frac{l_{i}^{n}+u_{i}^{n}}{2}, i=1, \ldots, k
$$

and step sizes

$$
c_{i}^{n}=\left(u_{i}^{n}-l_{i}^{n}\right) / 2, i=1, \ldots, k
$$

At the start of the algorithm, an initial starting point and initial step sizes should be given. Choosing the initial step sizes at the start of the algorithm should be done with extreme caution, as will discussed below.

\section{A. Approximate the simulation response func-} tion in the current region of interest by a firstorder model. The first-order model is given by

$$
y=\alpha_{0}+\sum_{i=1}^{k} \alpha_{i} \xi_{i}+\epsilon
$$

It is assumed that the additive error $\epsilon$ has a normal distribution with mean 0 and variance $\sigma^{2}$. To increase the computational accuracy in estimating the regression coefficients, the factors are coded, which gives the coded variables $x_{i}, i=1, \ldots, k$ :

$$
x_{i}=\frac{\xi_{i}-\xi_{i}^{n}}{c_{i}^{n}} \Longrightarrow \xi_{i}=c_{i}^{n} x_{i}+\xi_{i}^{n}
$$

and the coded first-order model:

$$
Y=\beta_{0}+\sum_{i=1}^{k} \beta_{i} x_{i}+\epsilon
$$

Estimators of the regression coefficients $\left\{\beta_{0}, \beta_{1}, \ldots, \beta_{k}\right\}$ are determined by using regression analysis and are denoted by $\left\{b_{0}, b_{1}, \ldots, b_{k}\right\}$. To this end, the objective function is evaluated in the points of an experimental design, which is a specific arrangement of points in the current region of interest. Although there are many designs to choose from, usually a fractional two-level factorial design of resolution-III (Kleijnen, 1998) is used, often augmented by the center point of the current region of interest (Myers and Montgomery, 1995). This design is orthogonal, which means that the variance of the predicted response in the region of interest is minimal, and that the regression coefficients can be assessed independently (Khuri and Cornell, 1996). Moreover, resolutionIII designs give unbiased estimators of the regression coefficients of a first-order polynomial (Kleijnen, 1998), and are efficient since the number of design points is small compared to other types of two-level factorial designs. Another advantage is that this type of design can quite easily be augmented to derive a second-order design. If the design is not within the domain $\mathrm{D}$, then it is moved into this region (Smith, 1976).

B. Test the first-order model for adequacy. Before using the first-order model to move into a direction of improved response, it should be tested if the estimated first-order model adequately describes the behaviour of the response in the current region of interest. If the true response shows interaction between the factors and / or pure curvature, the estimated firstorder model will likely show lack-of-fit, which can be assessed from the analysis of variance (ANOVA) table. Testing for lack of fit requires that the resolution-III design used for estimating the regression coefficients of the first-order model is not saturated, i.e. the total number of observations should be larger than the number of regression coefficients. Moreover, multiple observations are needed in the center point of the region of interest (Box and Draper, 1987). Furthermore, it could happen that although the first-order model does fit well, it is not possible to determine a direction of improved response from this model. This occurs when the estimated regression coefficients are not significantly different from zero, which can also be assessed using the ANOVA table.

At the start of the algorithm it should be decided which of these tests to use, i.e. when to accept the first-order model. For example, if there is interaction between the factors but no pure curvature, one could still decide to accept the first-order model. The decisions include choosing the significance levels for the tests involved.

C. Perform a line search in the steepest descent direction. If the first-order model is accepted, 
then it is used for determining the direction where improvement of the simulation response is expected. The steepest descent direction is given by $\left(-b_{1}, \ldots,-b_{k}\right)$. A line search is performed from the center point of the current region of interest in this direction to find a point of improved response. This point is taken as the estimator of the optimum of the simulation response function in the $n$th iteration and is used as the center point of the region of interest in the $(n+1)$ th iteration, i.e. $\left\{\xi_{1}^{n+1}, \ldots, \xi_{k}^{n+1}\right\}$.

In most RSM literature, line search is applied as follows (Box and Draper, 1987; Myers and Montgomery, 1995; Khuri and Cornell, 1996). First, increments $\left(\Delta_{1}, \ldots, \Delta_{k}\right)$ along the steepest descent direction are chosen, where $\Delta_{1} \div \ldots \div \Delta_{k}=b_{1} \div \ldots \div b_{k}$. These increments are usually determined by subjectively choosing a most important factor, e.g. $\xi_{j}$. This factor will be increased (decreased) by 1.0 unit in coded values, i.e. this factor will be increased by $c_{j}^{n}$. The other factors are consequently increased by

$$
\Delta_{i}=\frac{b_{i}}{b_{j}}, i=1, \ldots, k, i \neq j
$$

units in coded variables. Alternatively, one could objectively choose such a factor by determining $j$ such that $j=\arg \max _{i=1, \ldots, k}\left|b_{i}\right|$. Another option is to set the increments $\left(\Delta_{1}, \ldots, \Delta_{k}\right)$ equal to the distance from the center point to the point of intersection of the direction of steepest descent and the sphere given by $\sum_{i=1}^{k} \Delta_{i}^{2}=1$. (Neddermeijer et al., 1999). The $m$ th line search point is given by $\left\{\xi_{1}^{n}+m \Delta_{1} c_{1}^{n}, \ldots, \xi_{k}^{n}+m \Delta_{k} c_{k}^{n}\right\}$. It follows that the initial step sizes chosen at the start of the algorithm have a direct effect on the magnitude of the movement of the factors, whereas it has no effect on the direction of steepest descent (Myers and Montgomery, 1995). As soon as a boundary of the domain $\mathrm{D}$ is crossed, the line search is continued along the projection of the search direction on this boundary (Smith, 1976).

For ending this type of line search, a stopping rule has to be chosen. The usual recommendation in RSM is to stop the line search when no further improvement is observed (Del Castillo, 1997). The most straightforward rule ends the line search when an observed value of the simulation response function is higher than the preceding observation, i.e. set $\xi_{i}^{n+1}$ equal to line search point $m$ if line search point $m+1$ is the first line search point for which no improvement was found. This rule is sensitive to the noise from the response surface function. Therefore the new center point is probably not optimal. Del Castillo (1997) compares this stopping rule with a number of rules that do take the noise into account. These include two other empirical stopping rules, i.e. the 2-in-a-row and the 3-in-a-row stopping rules, that end the line search when 2 or 3 consecutive observed values of the simulation response function are higher than the preceding observation. In the Myers and Khuri stopping rule, the line search ends when an observed value of the simulation response function is significantly higher than the preceding observation. Del Castillo proposes a stopping rule with variable increments that is based on recursive estimation of secondorder polynomials along the search direction. Based on simulated line searches Del Castillo finds that both this recursive procedure and the Myers and Khuri rule perform better that the empirical stopping rules.

$\mathrm{Fu}$ (1994) describes another type of line search algorithms where a set of experiments along the steepest descent direction is performed. From these experiments, a one-dimensional second-order polynomial is estimated. This polynomial is optimized to derive the next center point $\xi_{i}^{n+1}$. Safizadeh and Signorile (1994) mention a similar line search algorithm. In addition, Joshi, Sherali and Tew (1998) introduce a line search algorithm which applies gradient deflection methods to prevent zigzagging of the steepest descent directions in multiple iterations.

\section{Solve the inadequacy of the first-order}

model. If the first-order model is not accepted, then either there is some evidence of pure curvature or interaction between the factors in the current region of interest, or the steepest descent direction cannot be discerned from zero. Usually, this is solved by approximating the simulation response function in the region of interest by a second-order polynomial. However, the optimization algorithm becomes less efficient especially if this occurs very early during the optimization exer- 
cise. Therefore, an alternative solution is to reduce the size of the region of interest by decreasing the step sizes $c_{i}^{n}, i=1, \ldots, k$. In this way this region can possibly become small enough to ensure that a first-order approximation is an adequate local representation of the simulation response function. Another solution is to increase the simulation size used in evaluating a design point or to increase the number of replicated observations done in the design points. This may ensure that indeed a significant direction of steepest descent is found.

At the start of the algorithm it should be decided which actions will be taken if the first-order model is rejected. Different actions can be taken depending on the outcome of the tests and the stage of the optimization exercise. For example, depending on the p-value found for the lack-of-fit test, one could decide to apply a second-order approximation or to decrease the size of the region of interest.

\section{E. Approximate the objective function in the} current region of interest using a second-order model. The coded second-order model is given by:

$$
Y=\beta_{0}+\sum_{i=1}^{k} \beta_{i} x_{i}+\sum_{i=1}^{k} \beta_{i, i} x_{i}^{2}+\sum_{i=1}^{k} \sum_{i<j}^{k} \beta_{i, j} x_{i} x_{j}+\epsilon
$$

The regression coefficients of the second-order model are again determined by using regression analysis applied to observations performed in an experimental design. The most popular class of second-order designs is the central composite design (CCD) (Myers and Montgomery, 1995). As was mentioned above, this design can easily be constructed by augmenting the fractional factorial design that was used for estimating the firstorder model. It is common to construct the CCD in such a way that it is rotatable, which means that the variance of the predicted response remains constant at all points which are equidistant to the center point of the current region of interest (Khuri and Cornell, 1996). Furthermore, the CCD can be transformed such that it is orthogonal by choosing a specific number of replicated observations in the center point of the current region of interest (Khuri and Cornell, 1996). If the design is not within the domain $\mathrm{D}$, then it is moved into this region (Smith, 1976).
F. Testing the second-order model for adequacy. Similar to the first-order model, it should be tested if the estimated second-order model adequately describes the behaviour of the response in the current region of interest before using this model. The secondorder model can be tested for the presence of lack-of-fit using the ANOVA table which results from the fitting procedure.

G. Solve the inadequacy of the second-order model. If the second-order model is found not to be adequate, then one can reduce the size of the region of interest (Joshi, Sherali and Tew, 1998) or increase the simulation size used in evaluating a design point. In RSM it is not customary to fit a higher than secondorder polynomial (Kleijnen, 1998).

H. Perform canonical analysis. If the secondorder model is found to be adequate, then canonical analysis is performed to determine the location and the nature of the stationary point of the second-order model. The estimated second-order approximation can be written as follows:

$$
\hat{Y}=\beta_{0}+\mathbf{x}^{\prime} \mathbf{b}+\mathbf{x}^{\prime} \mathbf{B} \mathbf{x}
$$

where

$$
\begin{aligned}
\mathbf{b}= & \left(b_{1}, \ldots, b_{k}\right) \\
\mathbf{B}= & \left(\begin{array}{llll}
b_{1,1} & b_{1,2} / 2 & \cdots & b_{1, k} / 2 \\
& b_{2,2} & \cdots & b_{2, k} / 2 \\
& & \ddots & \vdots \\
\text { sym. } & & & b_{k, k}
\end{array}\right)
\end{aligned}
$$

The stationary point $\mathbf{s}$ of the second-order polynomial is determined by

$$
\mathbf{s}=-\frac{1}{2} \mathbf{B}^{-1} \mathbf{b}
$$

Let $\mathbf{E}$ be the matrix of normalized eigenvectors of $\mathbf{B}$ and let $v_{1}, \ldots, v_{k}$ be the eigenvalues of $\mathbf{B}$. If all eigenvalues are positive (negative), then the quadratic surface has a minimum (maximum) at the stationary point $\mathbf{s}$. If the eigenvalues are mixed in sign, then the stationary point $\mathbf{s}$ is a saddle point.

I. Perform ridge analysis. It is not advisable to extrapolate the second-order polynomial beyond the 
current region of interest (Myers and Montgomery, 1995). Therefore, if the stationary point is a minimum which lies outside the current region of interest, the stationary point is not accepted as the center of the next region of interest. If the stationary point is a maximum or a saddle point, then the stationary point is rejected as well. In these cases, ridge analysis is performed, which means that we search for a new stationary point $\mathbf{s}_{R}$ on a given radius $R$ such that the second order model has a minimum at this stationary point (Myers and Montgomery, 1995). Using Lagrange analysis with multiplier $\mu$, this stationary point is given by

$$
(\mathbf{B}-\mu \mathbf{I}) \mathbf{s}_{R}=-\frac{1}{2} \mathbf{b}
$$

and it should hold that $\mu<\min _{i} v_{i}$ and $\sqrt{\mathbf{s}_{R}^{\prime} \mathbf{s}_{R}}=R$. We can write

$$
R^{2}=\mathbf{s}_{R}^{\prime} \mathbf{s}_{R}=\sum_{i=1}^{k}\left(\frac{\mathbf{e}_{i}^{\prime} \mathbf{b}}{2\left(v_{i}-\mu\right)}\right)^{2}
$$

where $\mathbf{e}_{i}$ is the eigenvector corresponding to the $\mathrm{i}^{t h}$ eigenvalue $v_{i}$.

A choice for the radius $R$ has to be made. For example, one could consider the radius of the circumscribed sphere of the region of interest, i.e. $R=\sqrt{2}$, which means that we have to find $\mu<\min _{i} v_{i}$ such that

$$
\sum_{i=1}^{k}\left(\frac{\mathbf{e}_{i}^{\prime} \mathbf{b}}{2\left(v_{i}-\mu\right)}\right)^{2}=2
$$

Standard numerical methods for finding the root of an equation can be used to determine $\mu$.

J. Accept the stationary point. The stationary point will be used as the center point of the next region of interest. It should be decided whether a first-order or a second-order model is used to approximate the simulation response surface in this region. These decisions can be made dependent on the results of the canonical analysis. For example, if a minimum was found, it could be useful to explore a region around this minimum with a new second-order approximation. On the other hand, if a maximum or a saddle point was found, the optimum could still be located far away from the current region of interest. In this case, approximating this region with a first-order model and consequently performing a line search would be preferable. Allowing this return to the first phase of the RSM algorithm is a powerful self-correction mechanism (Neddermeijer, 1999).

K. Determine a steepest descent direction from the second-order model. Joshi, Sherali and Tew (1998) introduced an enhanced RSM algorithm, in which they use the gradient of the second-order model in the center point of the current region and the results of the canonical analysis to determine a direction of steepest descent. Next, they perform a line search using this direction, resulting in a new center of a region of interest. In this region the simulation response surface will be approximated by a first-order model.

Stopping criterion. In RSM literature, it is often proposed to end the algorithm after fitting only one second order polynomial (Fu, 1994, Kleijnen, 1998). However, we do not recommend this strategy for two reasons. First of all, this strategy assumes that a minimum inside the current region is found, and therefore excludes the cases in which either a minimum outside the current region is found or a maximum or a saddle point is found. Furthermore, Greenwood, Rees and Siochi (1998) find that even for simple simulation response surfaces, first-order models can be inappropriate over a large percentage of the domain. Depending on the choices made in the algorithm, this means that the optimization can turn to the second-order phase quite early in the optimization exercise. Consequently, if the optimization algorithm ends after only one second-order approximation, it is likely that the best point of the optimization is located far from the optimum.

Therefore, we recommend ending the optimization exercise if either the estimated optimal simulation response value does not improve sufficiently anymore, if the region of interest becomes too small, or, in case there are budget constraints, if a fixed maximum number of evaluations have been performed. Next, a confidence interval about the response at the estimator for the optimum and the location of this estimator can be determined, see e.g. Carter et al. (1984).

We want to underline the fact that the Nelder and Mead simplex method is a local search method. No 
guarantee is given for finding the global optimum. Therefore, when optimizing a stochastic objective function, multistart using multiple starting points and / or multiple searches from the same starting point should be considered.

\section{CONCLUSION}

In this paper, we proposed a framework for optimization of simulation models using Response Surface Methodology. In existing RSM literature, this methodology is usually applied in a non-automated fashion, and much work is done on improving separate parts of RSM. This paper is the first attempt to define a clear, detailed and consistent RSM algorithm. The framework is especially useful for automated optimization, in which all the settings of the algorithm have to be chosen at the outset of the optimization process. Based on this framework, additional research can be done on comparing the different settings of the RSM algorithm for automated optimization of simulation models. Furthermore, the question how the RSM algorithm compares to other algorithms such as the Nelder and Mead simplex method and Simultaneous Perturbation Stochastic Approximation remains to be addressed.

\section{REFERENCES}

Box, G.E.P, and N.R. Draper. 1987. Empirical ModelBuilding and Response Surfaces. New York: John Wiley \& Sons.

Box, G.E.P. and P.Y.T. Liu. 1999. Statistics as a Catalyst to Learning by Scientific Method Part - An Example. Journal of Quality Technology 31(1): 1-15.

Carson, Y. and A. Maria. 1997. Simulation Optimization: Methods and Applications. In Proceedings of the 1997 Winter Simulation Conference, eds. S. Andradóttir, K.J. Healy, D.H. Withers and B.L. Nelson, 118-126. Piscataway, New Jersey: IEEE Press.

Carter W.H., V.M. Chinchilli, E.D. Campbell and G.L. Wampler. 1984. Confidence Intervals about the Response at the Stationary Point of a Response Surface, with an Application to Preclinical Cancer Therapy. Biometrics 40: 1125-1130.
Cochran W.G. and G.M. Cox. 1962. Experimental Designs. New York: John Wiley \& Sons.

Del Castillo, E. 1997. Stopping Rules for Steepest Ascent in Experimental Optimization. Communications in Statistics. Simulation and Computation 26(4): 1599-1615.

Fu, M.C. 1994. Optimization via Simulation: A Review. Annals of Operations Research 53: 199-247.

$\mathrm{Fu}$ M.C. and S.D. Hill. 1996. Optimization of Discrete Event Systems via Simultaneous Perturbation Stochastic Approximation. IIE Transactions 29: 233-243.

Greenwood, A.G., L.P. Rees and F.C. Siochi. 1998. An Investigation of the Behavior of Simulation Response Surfaces. European Journal of Operational Research 110: 282-313.

Hood S.J. and P.D. Welch. 1993. Response Surface Methodology and its Application in Simulation. In Proceedings of the 1993 Winter Simulation Conference, eds. G.W. Evans, M. Mollaghasemi, E.C. Russell and W.E. Biles, 115-122. Piscataway, New Jersey: IEEE Press.

Joshi, S., H.D. Sherali and J.D. Tew. 1998. An Enhanced Response Surface Methodology (RSM) Algorithm Using Gradient Deflection and Second-Order Search Strategies. Computers and Operations Research 25(7/8): 531-541.

Khuri, A.I. and J.A. Cornell. 1996. Response Surfaces: Designs and Analyses. New York: Marcel Dekker, Inc.

Kleijnen, J.P.C., 1998. Experimental Design for Sensitivity Analysis, Optimization, and Validation of Simulation models. In Handbook of Simulation: Principles, Methodology, Advances, Applications and Practice, ed. J. Banks, 173-223. New York: John Wiley $\&$ Sons.

Myers, R.H. and D.C. Montgomery. 1995. Response Surface Methodology: Process and Product Optimization Using Designed Experiments. New York: John Wiley \& Sons.

Neddermeijer, H.G., N. Piersma, G.J. van Oortmarssen, J.D.F. Habbema and R. Dekker. 1999. Comparison of Response Surface Methodology and 
the Nelder and Mead Simplex Method for Optimization in Microsimulation Models. Econometric Institute Report EI-9924/A, Erasmus University Rotterdam, The Netherlands.

Pflug, G.Ch. 1996. Optimization of Stochastic Models: The Interface Between Simulation and Optimization. Boston: Kluwer Academic Publishers.

Safizadeh, M.H. and R. Signorile. 1994. Optimization of Simulation via Quasi-Newton Methods. ORSA Journal on Computing 6(4): 398-408.

Smith, D.E. 1976. Automatic Optimum-Seeking program for Digital Simulation. Simulation 27: 27-31.

Wright, M.H. 1996. Direct Search Methods: Once Scorned, Now Respectable. In Numerical Analysis 1995: Proceedings of the 1995 Dundee Biennial Conference in Numerical Analysis., eds. D. F. Griffiths, and G. A. Watson, 191-208. Harlow, UK: Addison Wesley Longman.

\section{AUTHOR BIOGRAPHIES}

H. GONDA NEDDERMEIJER is a Ph.D. Candidate in the Econometric Institute and the Department of Public Health at the Erasmus University Rotterdam, The Netherlands. She received a M.Sc. degree in technical mathematics at the Delft University of Technology and a M.Sc. degree in Health Services Research at the Erasmus University Rotterdam. She is a member of INFORMS. Her research interests include optimization of stochastic simulation models and simulation models for screening for early detection of cancer. Her email and web addresses are <neddermeijer@few.eur.nl> and <www.few.eur.nl/few/people/neddermeijer>.

GERRIT J. VAN OORTMARSSEN is assistant professor of Public Health at the Department of Public Health of the Erasmus University Rotterdam. He obtained his Ph.D. at the Erasmus University, and his M.Sc. degree in applied mathematics from the Twente University of Technology. His research interest is (simulation) modeling of public health interventions for diseases, in particular applied to screening for early detection of cancer, and control of tropical in- fectious diseases (onchocerciasis, schistosomiasis, lymphatic filariasis, leprosy, STDs). His email address is <vanoortmarssen@mgz.fgg. eur.nl>.

NANDA PIERSMA is assistant professor in operations research at the Econometric Institute of the Erasmus University Rotterdam. She obtained her Ph.D. in operations research and her M.Sc. degree in mathematics at the University of Amsterdam. Her research interest is stochastic modeling in operations research and marketing econometrics. Her email and web addresses are <piersma@few.eur.nl> and <ww. few.eur.nl/few/people/piersma>.

ROMMERT DEKKER is a full professor in operations research at the Econometric Institute of the Erasmus University Rotterdam. He obtained his Ph.D. in operations research at the State University of Leiden and his M.Sc. degree in industrial engineering from the Twente University of Technology. He has worked for seven years with Shell. His current research interests are: maintenance and logistics (inventory control, spare parts, containers and reverse logistics). He has many times applied simulation models in various logistical activities. His email and web addresses are <rdekker@few.eur.nl> and $<$ ww. few.eur.nl/few/people/rdekker>. 